\title{
A generalization of Schönemann's theorem via a graph theoretic method
}

\author{
Khodakhast Bibak $^{*} \quad$ Bruce M. Kapron ${ }^{\dagger} \quad$ Venkatesh Srinivasan ${ }^{\ddagger}$ \\ May 7, 2019
}

\begin{abstract}
Recently, Grynkiewicz et al. [Israel J. Math. 193 (2013), 359-398], using tools from additive combinatorics and group theory, proved necessary and sufficient conditions under which the linear congruence $a_{1} x_{1}+\cdots+a_{k} x_{k} \equiv b(\bmod n)$, where $a_{1}, \ldots, a_{k}, b, n$ $(n \geq 1)$ are arbitrary integers, has a solution $\left\langle x_{1}, \ldots, x_{k}\right\rangle \in \mathbb{Z}_{n}^{k}$ with all $x_{i}$ distinct. So, it would be an interesting problem to give an explicit formula for the number of such solutions. Quite surprisingly, this problem was first considered, in a special case, by Schönemann almost two centuries ago(!) but his result seems to have been forgotten. Schönemann [J. Reine Angew. Math. 1839 (1839), 231-243] proved an explicit formula for the number of such solutions when $b=0, n=p$ a prime, and $\sum_{i=1}^{k} a_{i} \equiv 0(\bmod p)$ but $\sum_{i \in I} a_{i} \not \equiv 0(\bmod p)$ for all $\emptyset \neq I \varsubsetneqq\{1, \ldots, k\}$. In this paper, we generalize Schönemann's theorem using a result on the number of solutions of linear congruences due to D. N. Lehmer and also a result on graph enumeration. This seems to be a rather uncommon method in the area; besides, our proof technique or its modifications may be useful for dealing with other cases of this problem (or even the general case) or other relevant problems.
\end{abstract}

Keywords: Linear congruence; distinct coordinates; graph enumeration

2010 Mathematics Subject Classification: 11D79, 11P83, 05C30

\section{Introduction}

Throughout the paper, we use $\left(a_{1}, \ldots, a_{k}\right)$ to denote the greatest common divisor (gcd) of the integers $a_{1}, \ldots, a_{k}$, and write $\left\langle a_{1}, \ldots, a_{k}\right\rangle$ for an ordered $k$-tuple of integers. Let $a_{1}, \ldots, a_{k}, b, n \in \mathbb{Z}, n \geq 1$. A linear congruence in $k$ unknowns $x_{1}, \ldots, x_{k}$ is of the form

$$
a_{1} x_{1}+\cdots+a_{k} x_{k} \equiv b \quad(\bmod n)
$$

*Department of Computer Science and Software Engineering, Miami University, Oxford, Ohio, 45056, USA. Email: bibakk@miamioh.edu

${ }^{\dagger}$ Department of Computer Science, University of Victoria, Victoria, BC, Canada V8W 3P6. Email: bmkapron@uvic.ca

${ }^{\ddagger}$ Department of Computer Science, University of Victoria, Victoria, BC, Canada V8W 3P6. Email: srinivas@uvic.ca 
By a solution of (1.1), we mean an $\mathbf{x}=\left\langle x_{1}, \ldots, x_{k}\right\rangle \in \mathbb{Z}_{n}^{k}$ that satisfies (1.1). The following result, proved by D. N. Lehmer [14], gives the number of solutions of the above linear congruence:

Proposition 1.1. Let $a_{1}, \ldots, a_{k}, b, n \in \mathbb{Z}, n \geq 1$. The linear congruence $a_{1} x_{1}+\cdots+a_{k} x_{k} \equiv b$ $(\bmod n)$ has a solution $\left\langle x_{1}, \ldots, x_{k}\right\rangle \in \mathbb{Z}_{n}^{k}$ if and only if $\ell \mid b$, where $\ell=\left(a_{1}, \ldots, a_{k}, n\right)$. Furthermore, if this condition is satisfied, then there are $\ell n^{k-1}$ solutions.

Counting the number of solutions of the above congruence with some restrictions on the solutions is also a problem of great interest. As an important example, one can mention the restrictions $\left(x_{i}, n\right)=t_{i}(1 \leq i \leq k)$, where $t_{1}, \ldots, t_{k}$ are given positive divisors of $n$. The number of solutions of the linear congruences with the above restrictions, which were called restricted linear congruences in [6], was first considered by Rademacher [16] in 1925 and Brauer [8] in 1926, in the special case of $a_{i}=t_{i}=1(1 \leq i \leq k)$, and they proved the following nice formula for the number $N_{n}(k, b)$ of such solutions:

$$
N_{n}(k, b)=\frac{\varphi(n)^{k}}{n} \prod_{p|n, p| b}\left(1-\frac{(-1)^{k-1}}{(p-1)^{k-1}}\right) \prod_{p \mid n, p \nmid b}\left(1-\frac{(-1)^{k}}{(p-1)^{k}}\right),
$$

where $\varphi(n)$ is Euler's totient function and the products are taken over all prime divisors $p$ of $n$. Since then, this problem has been studied, in several other special cases, in many papers (very recently, it was studied in its 'most general case' in [6]) and has found very interesting applications in number theory, combinatorics, geometry, computer science, cryptography etc; see [3, 4, 6, 17, 9, 11] for a detailed discussion about this problem and a comprehensive list of references. Another restriction of potential interest is imposing the condition that all $x_{i}$ are distinct. Unlike the first problem, there seems to be very little published on the second problem. Recently, Grynkiewicz et al. [10], using tools from additive combinatorics and group theory, proved necessary and sufficient conditions under which the linear congruence $a_{1} x_{1}+\cdots+a_{k} x_{k} \equiv b(\bmod n)$, where $a_{1}, \ldots, a_{k}, b, n(n \geq 1)$ are arbitrary integers, has a solution $\left\langle x_{1}, \ldots, x_{k}\right\rangle \in \mathbb{Z}_{n}^{k}$ with all $x_{i}$ distinct; see also [1, 10] for connections to zero-sum theory and [5] for connections to coding theory. So, it would be an interesting problem to give an explicit formula for the number of such solutions. Quite surprisingly, this problem was first considered, in a special case, by Schönemann [17] almost two centuries ago(!) but his result seems to have been forgotten. Schönemann [17] proved the following result:

Theorem 1.2. Let $p$ be a prime, $a_{1}, \ldots, a_{k}$ be arbitrary integers, and $\sum_{i=1}^{k} a_{i} \equiv 0(\bmod p)$ but $\sum_{i \in I} a_{i} \not \equiv 0(\bmod p)$ for all $\emptyset \neq I \varsubsetneqq\{1, \ldots, k\}$. The number $N_{p}(k)$ of solutions $\left\langle x_{1}, \ldots, x_{k}\right\rangle \in \mathbb{Z}_{p}^{k}$ of the linear congruence $a_{1} x_{1}+\cdots+a_{k} x_{k} \equiv 0(\bmod p)$, with all $x_{i}$ distinct, is independent of the coefficients $a_{1}, \ldots, a_{k}$ and is equal to

$$
N_{p}(k)=(-1)^{k-1}(k-1) !(p-1)+(p-1) \cdots(p-k+1) .
$$

In this paper, we generalize Schönemann's theorem using Proposition 1.1 and a result on graph enumeration. This seems to be a rather uncommon method in the area; besides, our proof technique or its modifications may be useful for dealing with other cases of this problem (or even the general case) or other relevant problems. We state and prove our main result in the next section. 


\section{Main Result}

Our generalization of Schönemann's theorem is obtained via a graph theoretic method which may be also of independent interest. We need two formulas on graph enumeration (see Theorem 2.2 below). These formulas are in terms of the deformed exponential function which is a special case of the three variable Rogers-Ramanujan function defined below. These functions have interesting applications in combinatorics, complex analysis, functional differential equations, and statistical mechanics (see [2, 12, 13, 15, 18, 19] and the references therein).

Definition 2.1. The three variable Rogers-Ramanujan function is

$$
R(\alpha, \beta, q)=\sum_{m \geq 0} \frac{\alpha^{m} \beta_{2}^{(m)}}{(1+q)\left(1+q+q^{2}\right) \cdots\left(1+q+\cdots+q^{m-1}\right)} .
$$

Also, the deformed exponential function is

$$
F(\alpha, \beta)=R(\alpha, \beta, 1)=\sum_{m \geq 0} \frac{\alpha^{m} \beta_{2}^{(m)}}{m !} .
$$

Let $g(c, e, k)$ be the number of simple graphs with $c$ connected components, $e$ edges, and $k$ vertices labeled $1, \ldots, k$, and $g^{\prime}(e, k)$ be the number of simple connected graphs with $e$ edges and $k$ labeled vertices. Suppose that

$$
G(t, y, z)=\sum_{c, e, k} g(c, e, k) t^{c} y^{e} \frac{z^{k}}{k !}
$$

and

$$
C G(y, z)=\sum_{e, k} g^{\prime}(e, k) y^{e} \frac{z^{k}}{k !}
$$

Theorem 2.2. ([2, 20]) The generating functions for counting simple graphs and simple connected graphs satisfy, respectively,

$$
G(t, y, z)=F(z, 1+y)^{t}
$$

and

$$
C G(y, z)=\log F(z, 1+y),
$$

where $F$ is the deformed exponential function defined above.

Now, we are ready to state and prove our main result:

Theorem 2.3. Let $a_{1}, \ldots, a_{k}, b, n(n \geq 1)$ be arbitrary integers, and $\left(\sum_{i \in I} a_{i}, n\right)=1$ for all $\emptyset \neq I \varsubsetneqq\{1, \ldots, k\}$. The number $N_{n}\left(b ; a_{1}, \ldots, a_{k}\right)$ of solutions $\left\langle x_{1}, \ldots, x_{k}\right\rangle \in \mathbb{Z}_{n}^{k}$ of the linear 
congruence $a_{1} x_{1}+\cdots+a_{k} x_{k} \equiv b(\bmod n)$, with all $x_{i}$ distinct, is

$$
\begin{aligned}
& N_{n}\left(b ; a_{1}, \ldots, a_{k}\right) \\
& = \begin{cases}(-1)^{k}(k-1) !+(n-1) \cdots(n-k+1), & \text { if }\left(\sum_{i=1}^{k} a_{i}, n\right) \nmid b ; \\
(-1)^{k-1}(k-1) !\left(\left(\sum_{i=1}^{k} a_{i}, n\right)-1\right)+(n-1) \cdots(n-k+1), & \text { if }\left(\sum_{i=1}^{k} a_{i}, n\right) \mid b .\end{cases}
\end{aligned}
$$

Proof. Let $\left\langle x_{1}, \ldots, x_{k}\right\rangle \in \mathbb{Z}_{n}^{k}$ be a solution of the linear congruence $a_{1} x_{1}+\cdots+a_{k} x_{k} \equiv b$ $(\bmod n)$. Note that our desired solutions are those for which none of the $\left(\begin{array}{l}k \\ 2\end{array}\right)$ equalities $x_{u}=x_{v}, 1 \leq u<v \leq k$, holds. Let $T_{k}=\{\{u, v\}: 1 \leq u<v \leq k\}$. By the inclusionexclusion principle, the number of such solutions is

$$
N_{n}\left(b ; a_{1}, \ldots, a_{k}\right)=\sum_{e=0}^{\left(\begin{array}{c}
k \\
2
\end{array}\right)}(-1)^{e} \sum_{\substack{S \subseteq T_{k} \\
|S|=e}} N(S)
$$

where $N(S)$ is the number of solutions of the linear congruence with $x_{\alpha}=x_{\beta}$ for $\{\alpha, \beta\} \in S$.

Now, we need to calculate

$$
\sum_{\substack{S \subseteq T_{k} \\|S|=e}} N(S)
$$

In order to calculate $N(S)$, we construct the graph $G(S)$ on vertices $1, \ldots, k$ and edge set $S$. In calculating $N(S)$ we note that all vertices $i$ in a connected component of $G(S)$ correspond to the same $x_{i}$ in the linear congruence (by the definition of $N(S)$ ), and so we can simplify the linear congruence by grouping the $x_{i}$ which are equal to each other. This procedure eventually gives a new linear congruence in which the coefficients are of the form $\sum_{i \in I} a_{i}$, where $\emptyset \neq I \subseteq\{1, \ldots, k\}$, and the number of terms is equal to the number of connected components of $G(S)$. If $G(S)$ has $c>1$ connected components then since $\left(\sum_{i \in I} a_{i}, n\right)=1$ for all $\emptyset \neq I \varsubsetneqq\{1, \ldots, k\}$, by Proposition 1.1 we have $N(S)=n^{c-1}$. Also, if $G(S)$ is connected, that is, $c=1$ then $N(S)$ is the number of solutions of the linear congruence $\left(\sum_{i=1}^{k} a_{i}\right) x \equiv b(\bmod n)$, and so by Proposition 1.1, $N(S)$ in this case, we denote it by $A$, is equal to $\left(\sum_{i=1}^{k} a_{i}, n\right)$ if $\left(\sum_{i=1}^{k} a_{i}, n\right) \mid b$, and is equal to zero otherwise. Let $g(c, e, k)$ be the number of simple graphs with $c$ connected components, $e$ edges, and $k$ vertices labeled $1, \ldots, k$, and $g^{\prime}(e, k)$ be the number of simple connected graphs with $e$ edges and $k$ labeled 
vertices. Now, recalling (2.1), we get

$$
\begin{aligned}
N_{n}\left(b ; a_{1}, \ldots, a_{k}\right) & =\sum_{e=0}^{\substack{k \\
2})}(-1)^{e}\left(A g^{\prime}(e, k)+\sum_{c=2}^{k} n^{c-1} g(c, e, k)\right) \\
& =A \sum_{e=0}^{\left(\begin{array}{c}
k \\
2
\end{array}\right)}(-1)^{e} g^{\prime}(e, k)+\frac{1}{n} \sum_{e=0}^{\left(\begin{array}{c}
k \\
2
\end{array}\right)} \sum_{c=2}^{k}(-1)^{e} n^{c} g(c, e, k) \\
& =(A-1) \sum_{e=0}^{\left(\begin{array}{c}
k \\
2
\end{array}\right)}(-1)^{e} g^{\prime}(e, k)+\frac{1}{n} \sum_{e=0}^{\left(\begin{array}{c}
k \\
2
\end{array}\right)} \sum_{c=1}^{k}(-1)^{e} n^{c} g(c, e, k) .
\end{aligned}
$$

Now, in order to evaluate the latter expression, we use the two formulas mentioned in Theorem 2.2. In fact, by Theorem 2.2, we have

$$
\sum_{e, k}(-1)^{e} g^{\prime}(e, k) \frac{z^{k}}{k !}=\log F(z, 0),
$$

and

$$
\sum_{c, e, k}(-1)^{e} n^{c} g(c, e, k) \frac{z^{k}}{k !}=F(z, 0)^{n},
$$

where $F$ is the deformed exponential function. Note that $F(z, 0)=1+z$. Now, we have

$$
\sum_{e=0}^{\left(\begin{array}{c}
k \\
2
\end{array}\right)}(-1)^{e} g^{\prime}(e, k)=\text { the coefficient of } \frac{z^{k}}{k !} \text { in } \log (1+z), \text { which is equal to } \frac{k !(-1)^{k+1}}{k},
$$

and

$$
\sum_{e=0}^{\left(\begin{array}{c}
k \\
2
\end{array}\right)} \sum_{c=1}^{k}(-1)^{e} n^{c} g(c, e, k)=\text { the coefficient of } \frac{z^{k}}{k !} \text { in }(1+z)^{n} \text {, which is equal to } k !\left(\begin{array}{l}
n \\
k
\end{array}\right) \text {. }
$$

Consequently, the number $N_{n}\left(b ; a_{1}, \ldots, a_{k}\right)$ of solutions $\left\langle x_{1}, \ldots, x_{k}\right\rangle \in \mathbb{Z}_{n}^{k}$ of the linear congruence $a_{1} x_{1}+\cdots+a_{k} x_{k} \equiv b(\bmod n)$, with all $x_{i}$ distinct, is

$$
\begin{aligned}
& N_{n}\left(b ; a_{1}, \ldots, a_{k}\right)=\frac{(A-1) k !(-1)^{k+1}}{k}+\frac{k !\left(\begin{array}{c}
n \\
k
\end{array}\right)}{n} \\
& = \begin{cases}(-1)^{k}(k-1) !+(n-1) \cdots(n-k+1), & \text { if }\left(\sum_{i=1}^{k} a_{i}, n\right) \nmid b ; \\
(-1)^{k-1}(k-1) !\left(\left(\sum_{i=1}^{k} a_{i}, n\right)-1\right)+(n-1) \cdots(n-k+1), & \text { if }\left(\sum_{i=1}^{k} a_{i}, n\right) \mid b .\end{cases}
\end{aligned}
$$


Remark 2.4. Note that in Schönemann's theorem, $b$ is zero and $n$ is prime but in Theorem [2.3, both $b$ and $n$ are arbitrary.

It would be an interesting problem to see if the technique presented in this paper can be modified so that it covers the problem in its full generality. So, we pose the following question.

Problem 1. Let $a_{1}, \ldots, a_{k}, b, n(n \geq 1)$ be arbitrary integers. Give an explicit formula for the number of solutions $\left\langle x_{1}, \ldots, x_{k}\right\rangle \in \mathbb{Z}_{n}^{k}$ of the linear congruence $a_{1} x_{1}+\cdots+a_{k} x_{k} \equiv b$ $(\bmod n)$ with all $x_{i}$ distinct.

Such results would be interesting from several aspects. As we mentioned in the Introduction, the number of solutions of the linear congruence with the restrictions $\left(x_{i}, n\right)=t_{i}$ $(1 \leq i \leq k)$, where $t_{1}, \ldots, t_{k}$ are given positive divisors of $n$, has found very interesting applications in number theory, combinatorics, geometry, computer science, cryptography etc. Therefore, having an explicit formula for the number of solutions with all $x_{i}$ distinct may also lead to interesting applications in these or other directions. The problem may also have implications in zero-sum theory (see [1, 10]) and in coding theory (see [5]).

\section{Acknowledgements}

The authors are grateful to the anonymous referees for a careful reading of the paper and helpful comments.

\section{References}

[1] D. Adams and V. Ponomarenko, Distinct solution to a linear congruence, Involve 3 (2010), 341-344.

[2] F. Ardila, F. Castillo, and M. Henley, The arithmetic Tutte polynomials of the classical root systems, Int. Math. Res. Not. 2015 (2015), 3830-3877.

[3] K. Bibak, B. M. Kapron, and V. Srinivasan, Counting surface-kernel epimorphisms from a co-compact Fuchsian group to a cyclic group with motivations from string theory and QFT, Nuclear Phys. B 910 (2016), 712-723.

[4] K. Bibak, B. M. Kapron, and V. Srinivasan, On a restricted linear congruence, Int. J. Number Theory 12 (2016), 2167-2171.

[5] K. Bibak, B. M. Kapron, and V. Srinivasan, Unweighted linear congruences with distinct coordinates and the Varshamov-Tenengolts codes, Des. Codes Cryptogr. 86 (2018), 1893-1904.

[6] K. Bibak, B. M. Kapron, V. Srinivasan, R. Tauraso, and L. Tóth, Restricted linear congruences, J. Number Theory 171 (2017), 128-144. 
[7] K. Bibak, B. M. Kapron, V. Srinivasan, and L. Tóth, On an almost-universal hash function family with applications to authentication and secrecy codes, Internat. J. Found. Comput. Sci. 29 (2018), 357-375.

[8] A. Brauer, Lösung der Aufgabe 30, Jber. Deutsch. Math.-Verein 35 (1926), 92-94.

[9] E. Cohen, A class of arithmetical functions, Proc. Natl. Acad. Sci. USA 41 (1955), 939-944.

[10] D. J. Grynkiewicz, A. Philipp, and V. Ponomarenko, Arithmetic-progression-weighted subsequence sums, Israel J. Math. 193 (2013), 359-398.

[11] D. Jacobson and K. S. Williams, On the number of distinguished representations of a group element, Duke Math. J. 39 (1972), 521-527.

[12] V. P. Kostov and B. Shapiro, Hardy-Petrovitch-Hutchinson's problem and partial theta function, Duke Math. J. 162 (2013), 825-861.

[13] J. K. Langley, A certain functional-differential equation, J. Math. Anal. Appl. 244 (2000), 564-567.

[14] D. N. Lehmer, Certain theorems in the theory of quadratic residues, Amer. Math. Monthly 20 (1913), 151-157.

[15] Y. Liu, On some conjectures by Morris et al., about zeros of an entire function, J. Math. Anal. Appl. 226 (1998), 1-5.

[16] H. Rademacher, Aufgabe 30, Jber. Deutsch. Math.-Verein 34 (1925), 158.

[17] T. Schönemann, Theorie der symmetrischen Functionen der Wurzeln einer Gleichung. Allgemeine Sätze über Congruenzen nebst einigen Anwendungen derselben, J. Reine Angew. Math. 1839 (1839), 231-243.

[18] A. D. Scott and A. D. Sokal, The repulsive lattice gas, the independent-set polynomial, and the Lovász local lemma, J. Stat. Phys. 118 (2005), 1151-1261.

[19] A. D. Sokal, The leading root of the partial theta function, Adv. Math. 229 (2012), 2603-2621.

[20] R. P. Stanley, Enumerative Combinatorics, Vol. 2, Cambridge University Press, (1999). 